\title{
Some Notes on Basic Syntactic Mutation $]^{*}$
}

\author{
Christopher Bouchard, Kimberly A. Gero, and Paliath Narendran \\ University at Albany-SUNY (USA) \\ \{cbou, kgero001, dran\}@cs.albany . edu
}

\begin{abstract}
Unification modulo convergent term rewrite systems is an important research area with many applications. In their seminal paper Lynch and Morawska gave three conditions on rewrite systems that guarantee that unifiability can be checked in polynomial time $(\mathbf{P})$. We show that these conditions are tight, in the sense that relaxing any one of them will "upset the applecart," giving rise to unification problems that are not in $\mathbf{P}$ (unless $\mathbf{P}=\mathbf{N P}$ ), and in doing so address an open problem posed by Lynch and Morawska. We also investigate a related decision problem: we show the undecidability of subterm-collapse for the restricted term rewriting systems that we are considering.
\end{abstract}

Keywords: Equational unification, Term rewriting, Polynomial-time complexity, NP-completeness, Undecidability.

\section{Introduction}

Unification modulo an equational theory $E$ (equational unification or $E$-unification) is an undecidable problem in general. Even in cases where it is decidable, it is often of high complexity. In their seminal paper "Basic Syntactic Mutation" [7] Christopher Lynch and Barbara Morawska present syntactic criteria on equational axioms $E$ that guarantee a polynomial time algorithm for the corresponding $E$-unification problem. As far as we know these are the only purely syntactic criteria that ensure a polynomial-time algorithm for unifiability. Our goal initially was to extend the Lynch-Morawska result for convergent term rewriting systems by relaxing their constraints, while still maintaining the polynomial time algorithm guarantee. However, we observed that their constraints were tight in the sense that relaxing any one of them would give rise to unification problems that are not in $\mathbf{P}$ (unless $\mathbf{P}=\mathbf{N P}$ ). We provide proofs that removing any of their constraints will lead to unification problems that are not in $\mathbf{P}$.

We also investigate one of the computational issues raised by the Lynch-Morawska paper, namely, checking whether a convergent term rewriting system is subterm-collapsing - a term rewriting system is subterm-collapsing if and only if there is a term that is congruent to a proper subterm of itself. We show the undecidability of subterm collapse even for convergent term rewriting systems that satisfy the Lynch-Morawska conditions [7]. (For general convergent systems, this result was shown by Bürkert, Herold and Schmidt-Schauß 4. 4 .)

\section{Notation and Preliminaries}

We assume the reader is familiar with the usual notions and concepts in term rewriting systems [2] and equational unification [3]. We consider rewrite systems over ranked signatures, usually denoted $\Sigma$, and a possibly infinite set of variables, usually denoted $\mathcal{X}$. The set of all

*Research supported in part by NSF grant CNS-0905286. 
terms over $\Sigma$ and $\mathcal{X}$ is denoted as $T(\Sigma, \mathcal{X})$. An equation is an ordered pair of terms $(s, t)$, usually written as $s \approx t$. Here $s$ is the left-hand side and $t$ is the right-hand side of the equation [2]. A rewrite rule is an equation $s \approx t$ where $\operatorname{Var}(t) \subseteq \operatorname{Var}(s)$, usually written as $s \rightarrow t$. A term rewriting system is a set of rewrite rules.

A set of equations $E$ is subterm-collapsing 1 if and only if there are terms $t$ and $u$ such that $t$ is a proper subterm of $u$ and $E \vdash t \approx u\left(\right.$ or $\left.t=_{E} u\right)$ [4. A set of equations $E$ is variablepreserving ${ }^{2}$ if and only if for every equation $t \approx u$ in $E, \mathcal{V} a r(t)=\mathcal{V}$ ar $(u)$ [9]. A term rewriting system is convergent if and only if it is confluent and terminating [2].

Given a set of equations $E$, the set of ground instances of $E$ is denoted by $\operatorname{Gr}(E)$. We assume a reduction order $\prec$ on $E$ which is total on ground terms. We extend this order to equations as $(s \approx t) \prec(u \approx v)$ iff $\{s, t\} \prec_{m u l}\{u, v\}$, where $\prec_{m u l}$ is the multiset order induced by $\prec$. An equation $e$ is redundant in $E$ if and only if every ground instance $\sigma(e)$ of $e$ is a consequence of equations in $\operatorname{Gr}(E)$ which are smaller than $\sigma(e)$ modulo $\prec$ [7].

\subsection{Paramodulation}

Lynch and Morawska define an extension to the critical pair rule called paramodulation. Since our focus is only on convergent term rewriting systems, this definition can be modified to use rewrite rules as

$$
\frac{u\left[s^{\prime}\right]_{p} \approx v \quad s \rightarrow t}{\sigma\left(u[t]_{p}\right) \approx \sigma(v)}
$$

where $\sigma=m g u\left(s=? s^{\prime}\right)$ and $p \in \mathcal{F P} \operatorname{os}(u)$. A set of equations $E$ is saturated if all inferences among equations in $E$ are redundant.

\subsection{Right-Hand-Side Critical Pairs}

Lynch and Morawska also define a right-hand-side critical pair. Again, we modify the definition to use rewrite rules as

$$
\frac{s \rightarrow t \quad u \rightarrow v}{\sigma(s) \approx \sigma(u)}
$$

where $\sigma=\operatorname{mgu}(v=? t)$ and $\sigma(s) \neq \sigma(u)$. For an equational theory $E$, we define $R H S(E)$ as follows [7:

$$
\begin{aligned}
R H S(E)=E \cup\{e \mid e \text { is the conclusion of a Right-Hand-Side Critical Pair } \\
\text { inference of two members of } E\}
\end{aligned}
$$

\subsection{Quasi-determinism}

A set of equations $E$ is quasi-deterministic if and only if

(1) No equation in $E$ has a variable as its left-hand side or right-hand side,

(2) No equation in $E$ is root-stable - i.e., no equation has the same root symbol on its leftand right-hand side, and

(3) $E$ has no root pair repetitions - i.e., no two equations in $E$ have the same pair of root symbols on their sides.

\footnotetext{
${ }^{1}$ Non-subterm-collapsing theories are called simple theories in 4

${ }^{2}$ Variable-preserving theories are also called non-erasing or regular theories [2].
} 
A theory $E$ is deterministic if and only if it is quasi-deterministic and non-subterm-collapsing.

Lemma 2.1. Suppose $R$ is a variable-preserving convergent rewrite system and $R$ is quasideterministic. Then $R H S(R)$ is not quasi-deterministic if and only if $R H S(R)$ has a root pair repetition.

Proof. If $R H S(R)$ has a root pair repetition, then clearly $R H S(R)$ is not quasi-deterministic by definition. We will prove the 'only if' case by contradiction.

Suppose $R H S(R)$ is not quasi-deterministic and has no root pair repetition. Since $R$ itself is quasi-deterministic, there must be equations created in $R H S(R)$ that cause the non-quasideterminism. Therefore there must be rules $l_{1} \rightarrow r_{1}$ and $l_{2} \rightarrow r_{2}$ in $R$ such that $\theta\left(l_{1}\right) \approx \theta\left(l_{2}\right)$ is in $R H S(R)$. Since $R$ is variable-preserving, it must be that $\left(l_{1} \rightarrow r_{1}\right)$ and $\left(l_{2} \rightarrow r_{2}\right)$ are distinct rules, for otherwise we would have $\theta\left(l_{1}\right)=\theta\left(l_{2}\right)$ and the generated rule would be discarded.

Since, by assumption, $R H S(R)$ has no root pair repetition, either one of $l_{1}$ or $l_{2}$ is a variable, or $\theta\left(l_{1}\right) \approx \theta\left(l_{2}\right)$ is root-stable. The first is not possible because rewrite rules cannot have a variable as their left-hand side. In the second case, rules $l_{1} \rightarrow r_{1}$ and $l_{2} \rightarrow r_{2}$ would cause a root pair repetition in $R$, which is a contradiction.

\subsection{Monotone 1-in-3-SAT}

All of the NP-completeness proofs in this paper are shown by reductions from the monotone 1-in-3-SAT problem [2], which is known to be NP-complete [6]. The problem is defined as follows:

Instance: A CNF formula $\mathcal{F}=C_{1} \wedge \cdots \wedge C_{n}$, where each clause is of the form $C_{i}=p_{i} \vee q_{i} \vee r_{i}$ and $p_{i}, q_{i}$, and $r_{i}$ are propositional variables.

Question: Is there a satisfying assignment to the propositional variables of $\mathcal{F}$ that sets exactly one propositional variable of each clause to true and the other two to false?

\section{Lynch-Morawska Conditions}

Given a confluent and terminating term rewriting system $R$, there are three conditions that must hold to maintain a polynomial time algorithm guarantee:

(1) $R$ is non-subterm-collapsing,

(2) $R$ is saturated by paramodulation, and

(3) $R H S(R)$ is quasi-deterministic.

In this section we will show that if any one of these conditions is relaxed there is no longer a polynomial time guarantee. Therefore the conditions given in the Lynch-Morawska paper are tight.

\section{1 $R$ is Subterm-Collapsing}

Consider the following single-rule ground term rewriting system $R_{1}$, where 0,1 , and $c$ are constants.

$$
f(0, f(0, f(1, c))) \rightarrow c
$$


The system $R_{1}$ is saturated by paramodulation and $R H S\left(R_{1}\right)$ is quasi-deterministic. However, it is subterm-collapsing. The unification problem can be shown to be NP-hard by a reduction from monotone 1-in-3-SAT.

For each clause $C_{i}=p_{i} \vee q_{i} \vee r_{i}$ we form the following equation $\mathcal{E} \mathcal{Q}_{i}$, where $Z_{i}$ is a variable that varies with each clause.

$$
f\left(V_{p_{i}}, f\left(V_{q_{i}}, f\left(V_{r_{i}}, Z_{i}\right)\right)\right)=\stackrel{?}{R_{1}} Z_{i}
$$

The unification problem $S$ is the set of all these equations. Every unifier of this equation replaces exactly one of $V_{p_{i}}, V_{q_{i}}$, or $V_{r_{i}}$ by 1 and the others by 0 . In fact,

$$
\begin{aligned}
\sigma_{1} & =\left\{V_{p_{i}} \mapsto 0, V_{q_{i}} \mapsto 0, V_{r_{i}} \mapsto 1, Z_{i} \mapsto c\right\} \\
\sigma_{2} & =\left\{V_{p_{i}} \mapsto 1, V_{q_{i}} \mapsto 0, V_{r_{i}} \mapsto 0, Z_{i} \mapsto f(1, c)\right\} \\
\sigma_{3} & =\left\{V_{p_{i}} \mapsto 0, V_{q_{i}} \mapsto 1, V_{r_{i}} \mapsto 0, Z_{i} \mapsto f(0, f(1, c))\right\}
\end{aligned}
$$

are the only normalized unifiers for the above equation. Clearly then $S$ is unifiable iff the 1-in-3-SAT instance has a solution.

In [7, Lynch and Morawska posed, as an open problem, whether the requirement that $R$ be non-subterm-collapsing can be removed; in other words, can the polynomial-time result be extended to systems which are saturated by paramodulation and quasi-deterministic but possibly subterm-collapsing? The rewrite system $R_{1}$ answers this problem negatively.

\section{2 $R$ is not Saturated by Paramodulation}

Consider the following rewrite system $R_{2}$, where 0 and 1 are constants.

$$
\begin{array}{ll}
f_{1}(s(x)) \rightarrow f_{2}(x) & f_{1}(0) \rightarrow g(0,0,1) \\
f_{2}(s(x)) \rightarrow f_{3}(x) & f_{2}(0) \rightarrow g(0,1,0) \\
& f_{3}(0) \rightarrow g(1,0,0)
\end{array}
$$

The system $R_{2}$ is deterministic (i.e., quasi-deterministic and non-subterm-collapsing), but not saturated by paramodulation. There are no right-hand-side critical pairs, so $\operatorname{RHS}\left(R_{2}\right)=R_{2}$. The unification problem is NP-hard by a reduction from monotone 1-in-3-SAT. We only present the key idea here.

For each propositional variable $p$ we form a respective term variable $V_{p}$. For each clause $C_{i}=p_{i} \vee q_{i} \vee r_{i}$ we form the following equation $\mathcal{E} \mathcal{Q}_{i}$.

$$
f_{1}\left(X_{i}\right)={ }_{R_{2}}^{?} g\left(V_{p_{i}}, V_{q_{i}}, V_{r_{i}}\right)
$$

It is not hard to see that the only unifiers of $\mathcal{E} \mathcal{Q}_{i}$ are:

$$
\begin{aligned}
& \sigma_{1}=\left\{V_{p_{i}} \mapsto 0, V_{q_{i}} \mapsto 0, V_{r_{i}} \mapsto 1, X_{i} \mapsto 0\right\} \\
& \sigma_{2}=\left\{V_{p_{i}} \mapsto 0, V_{q_{i}} \mapsto 1, V_{r_{i}} \mapsto 0, X_{i} \mapsto s(0)\right\} \\
& \sigma_{3}=\left\{V_{p_{i}} \mapsto 1, V_{q_{i}} \mapsto 0, V_{r_{i}} \mapsto 0, X_{i} \mapsto s(s(0))\right\}
\end{aligned}
$$

In the case where $R$ is not saturated by paramodulation, there is even a rewrite system whose unification problem is not decidable. Consider the following single-rule rewriting system $R_{2}^{\prime}$, where $B$ and $*$ are binary function symbols.

$$
B(x, y) * B(u, v) \rightarrow B(x * u, y * v)
$$

The system $R_{2}^{\prime}$ is also deterministic and not saturated by paramodulation. Again, there are no right-hand-side critical pairs, so $R H S\left(R_{2}^{\prime}\right)=R_{2}^{\prime}$. The unification problem modulo $R_{2}^{\prime}$ is undecidable [1]. 


\section{3 $R H S(R)$ is not Quasi-deterministic}

By Lemma 2.1, there are only two ways $R H S(R)$ can be non-quasi-deterministic: either $R$ itself is not quasi-deterministic, or $R H S(R)$ contains a root pair repetition. Furthermore, the only way that $R$ can be non-quasi-deterministic (other than a root pair repetition) is if it contains a root-stable equation. This is because a rewrite rule cannot have a variable as its left-hand side, and a rule with a variable as its right-hand side would make the system subterm-collapsing.

\section{$R$ has a Root-stable Equation}

The following system $R_{3}$ is non-subterm-collapsing and saturated by paramodulation. However $R_{3}$ is not quasi-deterministic because it contains root-stable equations.

$$
f(g(0,0,1), f(g(0,1,0), c)) \rightarrow f(g(1,0,0), f(g(1,0,0), c))
$$

where $c, 0$, and 1 are constants. Unifiability modulo $R_{3}$ can also be shown to be NP-hard by a reduction from monotone 1-in-3-SAT. Again, we only present the key idea here.

For each propositional variable $p$ we form a respective term variable $V_{p}$. For each clause $C_{i}=p_{i} \vee q_{i} \vee r_{i}$ we form the following equation $\mathcal{E} \mathcal{Q}_{i}$, where $X_{i}, Y_{i}$, and $Z_{i}$ are variables that vary with each clause.

$$
f\left(X_{i}, f\left(g\left(V_{p_{i}}, V_{q_{i}}, V_{r_{i}}\right), Y_{i}\right)\right)={ }_{R_{3}}^{?} f\left(g(1,0,0), f\left(g(1,0,0), Z_{i}\right)\right)
$$

To unify this equation, we can either unify syntactically, or we can apply the rewrite rule to the left-hand side at either occurrence of $f$. The following is a complete set of unifiers of $\mathcal{E} \mathcal{Q}_{i}$ :

$$
\begin{aligned}
\sigma_{1}= & \left\{V_{p_{i}} \mapsto 1, V_{q_{i}} \mapsto 0, V_{r_{i}} \mapsto 0, X_{i} \mapsto g(1,0,0), Y_{i} \mapsto Z_{i}\right\} \\
\sigma_{2}= & \left\{V_{p_{i}} \mapsto 0, V_{q_{i}} \mapsto 1, V_{r_{i}} \mapsto 0, X_{i} \mapsto g(0,0,1), Y_{i} \mapsto c, Z_{i} \mapsto c\right\} \\
\sigma_{3}= & \left\{V_{p_{i}} \mapsto 0, V_{q_{i}} \mapsto 0, V_{r_{i}} \mapsto 1, X_{i} \mapsto g(1,0,0), Y_{i} \mapsto f(g(0,1,0), c),\right. \\
& \left.Z_{i} \mapsto f(g(1,0,0), c)\right\}
\end{aligned}
$$

Note that every unifier replaces exactly one of $V_{p_{i}}, V_{q_{i}}$, or $V_{r_{i}}$ by 1 and the others by 0 .

\section{$R H S(R)$ has a Root Pair Repetition}

The following system $R_{4}$ is non-subterm-collapsing and saturated by paramodulation. However $R H S\left(R_{4}\right)$ is not quasi-deterministic because it has a root pair repetition.

$$
\begin{array}{ll}
f(0,0,1) \rightarrow c_{1} & g(0,0,1) \rightarrow c_{1} \\
f(0,1,0) \rightarrow c_{2} & g(0,1,0) \rightarrow c_{2} \\
f(1,0,0) \rightarrow c_{3} & g(1,0,0) \rightarrow c_{3}
\end{array}
$$

where $c_{1}, c_{2}, c_{3}, 0$, and 1 are constants. Note that $R_{4}$ is deterministic. Unifiability modulo $R_{4}$ can also be shown to be NP-hard by a reduction from monotone 1-in-3-SAT. Again, we only present the key idea here.

For each propositional variable $p$ we form a respective term variable $V_{p}$. For each clause $C_{i}=p_{i} \vee q_{i} \vee r_{i}$ we form the following equation $\mathcal{E} \mathcal{Q}_{i}$.

$$
f\left(V_{p_{i}}, V_{q_{i}}, V_{r_{i}}\right)={ }_{R_{4}}^{?} g\left(V_{p_{i}}, V_{q_{i}}, V_{r_{i}}\right)
$$


The only unifiers of $\mathcal{E} \mathcal{Q}_{i}$ are:

$$
\begin{aligned}
& \sigma_{1}=\left\{V_{p_{i}} \mapsto 0, V_{q_{i}} \mapsto 0, V_{r_{i}} \mapsto 1\right\} \\
& \sigma_{2}=\left\{V_{p_{i}} \mapsto 0, V_{q_{i}} \mapsto 1, V_{r_{i}} \mapsto 0\right\} \\
& \sigma_{3}=\left\{V_{p_{i}} \mapsto 1, V_{q_{i}} \mapsto 0, V_{r_{i}} \mapsto 0\right\}
\end{aligned}
$$

\section{Undecidability of Subterm-Collapse}

For general convergent systems, this result was shown by Bürkert, Herold and SchmidtSchauß 4. We show that the property of subterm-collapsing is undecidable even when the convergent system $R$ satisfies the other Lynch-Morawska conditions, namely saturation by paramodulation and quasi-determinism of $R H S(R)$.

The proof is by reduction from the halting problem for reversible deterministic 2-counter Minsky machines, which is undecidable since reversible deterministic 2-counter Minsky machines are Turing-universal [8]. Such a machine $M$ has two counters $C_{1}$ and $C_{2}$ and is described by a quadruple $\left(Q, \delta, q_{0}, q_{L}\right)$, where $Q=\left\{q_{0}, \ldots, q_{L}\right\}$ is the set of states, $q_{0}$ is the initial state, $q_{L}$ is the final state, and $\delta$ is the move relation. The relation $\delta$ is represented by quadruples of the form

$$
\left[q_{i}, j, s, q_{k}\right] \text { or }\left[q_{i}, j, d, q_{k}\right]
$$

where $q_{i}, q_{k} \in Q, j=1$ or $2, s \in\{Z, P\}$, and $d \in\{-, 0,+\}$. The first form represents checking if counter $C_{j}$ is zero $(Z)$ or positive $(P)$ and if so moving to state $q_{k}$. The second form represents decrementing $(-)$, incrementing $(+)$, or doing nothing to $(0)$ the counter $C_{j}$ and then moving to state $q_{k}$.

We assume, without loss of generality, that $\delta$ does not contain any quadruples with $q_{0}$ as the destination. We can assume this because a machine with such a move relation can be transformed into a machine $M^{\prime}=\left(Q \uplus\left\{q_{0}^{\prime}\right\}, \delta^{\prime}, q_{0}^{\prime}, q_{L}\right)$ where $\delta^{\prime}=\delta \uplus\left\{\left[q_{0}^{\prime}, 1,0, q_{0}\right]\right\}$. This new machine is clearly computationally equivalent to $M$.

We represent the configuration of such machine with a triple $\left(q_{i}, C_{1}, C_{2}\right)$, where $q_{i}$ is the current state of the machine, and $C_{1}$ and $C_{2}$ are the states (values) of the counters. The machine is assumed to be deterministic and reversible, which means that any configuration has at most one predecessor. By Definition 2.3 of [ 8 , a machine $M$ is deterministic and reversible if and only if, for each pair of quadruples $\left[q_{i_{1}}, j_{1}, x_{1}, q_{i_{1}^{\prime}}\right]$ and $\left[q_{i_{2}}, j_{2}, x_{2}, q_{i_{2}^{\prime}}\right]$ in $\delta$,

$$
\left(i_{1}=i_{2} \text { or } i_{1}^{\prime}=i_{2}^{\prime}\right) \Rightarrow\left(j_{1}=j_{2} \text { and }\left\{x_{1}, x_{2}\right\}=\{Z, P\}\right)
$$

Thus each state has at most two predecessors and two successors, but only if the state is entered and left (respectively) by checking the state of a single counter.

Given such a machine $M$ and configurations $\left(q_{0}, k, p\right)$ and $\left(q_{L}, k^{\prime}, p^{\prime}\right)$, we construct a term rewriting system $R_{M}$ such that $R_{M}$ is saturated by paramodulation, and $R H S\left(R_{M}\right)$ is quasideterministic. We then show that $R_{M}$ is subterm-collapsing if and only if the machine $M$, starting in configuration $\left(q_{0}, k, p\right)$, will halt with $\left(q_{L}, k^{\prime}, p^{\prime}\right)$ as its final configuration.

Our system is over the signature $\Sigma=\bigcup_{i=0}^{L}\left\{q_{i}, f_{i}, f_{i}^{\prime}\right\} \cup\left\{0, e, e^{\prime}, c, s, f, g, g^{\prime}\right\}$ where $0, e, e^{\prime}$, and $q_{0}, \ldots, q_{L}$ are constants, $c$ has arity 4 , every other symbol has arity 1.

We can encode a natural number $n$ as a term $s^{n}(0)$, where $s$ represents the successor function and 0 represents the natural number 0 . Each state $q_{i}$ will encode itself as a constant. We use $e$ and $e^{\prime}$ to represent the 'empty' state, which means the machine has either not started or has already halted. 
We can then encode a configuration $\left(q_{i}, k, p\right)$ as a term $c\left(q_{i}, s^{k}(0), s^{p}(0), s^{n}(0)\right)$, where $n$ is the number of steps the machine has taken. The symbol $c$ acts only as a constructor. We use the $f$ and $g$ symbols, with various subscripts and primes, to ensure termination and saturation by paramodulation of the resulting rewrite system.

To start, initialize $R_{M}$ to the following rewrite system:

$$
\begin{aligned}
f\left(c\left(e, s^{k}(0), s^{p}(0), 0\right)\right) & \rightarrow c\left(q_{0}, s^{k}(0), s^{p}(0), 0\right) \\
f_{L}\left(c\left(q_{L}, s^{k^{\prime}}(0), s^{p^{\prime}}(0), z\right)\right) & \rightarrow g\left(c\left(e^{\prime}, 0,0, z\right)\right) \\
g^{\prime}\left(g\left(c\left(e^{\prime}, 0,0, s(z)\right)\right)\right) & \rightarrow c\left(e^{\prime}, 0,0, z\right) \\
g^{\prime}\left(g\left(c\left(e^{\prime}, 0,0,0\right)\right)\right) & \rightarrow e
\end{aligned}
$$

The first rule encodes initializing the machine, moving from an empty state $e$ to the initial state $q_{0}$ iff the configuration matches the given starting configuration. The second rule terminates the machine iff the configuration matches the given final configuration and moves to an empty state $e^{\prime}$. The third rule checks that the fourth argument in the $c$-term encodes a valid natural number. The last rule takes a correctly terminated machine and gives the empty state, which could cause a subterm-collapse.

Next we encode the transition rules. For each quadruple in $\delta$, extend $R_{M}$ using one of the following transformations:

$$
\begin{aligned}
& \text { (a1) }\left[q_{i}, 1, P, q_{j}\right]: R_{M}:=R_{M} \cup\left\{f_{i}\left(c\left(q_{i}, s(x), y, z\right)\right) \rightarrow c\left(q_{j}, s(x), y, s(z)\right)\right\} \\
& \text { (a2) }\left[q_{i}, 2, P, q_{j}\right]: R_{M}:=R_{M} \cup\left\{f_{i}\left(c\left(q_{i}, x, s(y), z\right)\right) \rightarrow c\left(q_{j}, x, s(y), s(z)\right)\right\} \\
& \text { (b1) }\left[q_{i}, 1, Z, q_{j}\right]: R_{M}:=R_{M} \cup\left\{f_{i}^{\prime}\left(c\left(q_{i}, 0, y, z\right)\right) \rightarrow c\left(q_{j}, 0, y, s(z)\right)\right\} \\
& \text { (b2) }\left[q_{i}, 2, Z, q_{j}\right]: R_{M}:=R_{M} \cup\left\{f_{i}^{\prime}\left(c\left(q_{i}, x, 0, z\right)\right) \rightarrow c\left(q_{j}, x, 0, s(z)\right)\right\} \\
& \text { (c1) }\left[q_{i}, 1,+, q_{j}\right]: R_{M}:=R_{M} \cup\left\{f_{i}\left(c\left(q_{i}, x, y, z\right)\right) \rightarrow c\left(q_{j}, s(x), y, s(z)\right)\right\} \\
& \text { (c2) }\left[q_{i}, 2,+, q_{j}\right]: R_{M}:=R_{M} \cup\left\{f_{i}\left(c\left(q_{i}, x, y, z\right)\right) \rightarrow c\left(q_{j}, x, s(y), s(z)\right)\right\} \\
& \text { (d1) }\left[q_{i}, 1,0, q_{j}\right]: R_{M}:=R_{M} \cup\left\{f_{i}\left(c\left(q_{i}, x, y, z\right)\right) \rightarrow c\left(q_{j}, x, y, s(z)\right)\right\} \\
& \text { (d2) }\left[q_{i}, 2,0, q_{j}\right]: R_{M}:=R_{M} \cup\left\{f_{i}\left(c\left(q_{i}, x, y, z\right)\right) \rightarrow c\left(q_{j}, x, y, s(z)\right)\right\} \\
& \text { (e1) }\left[q_{i}, 1,-, q_{j}\right]: R_{M}:=R_{M} \cup\left\{f_{i}\left(c\left(q_{i}, s(x), y, z\right)\right) \rightarrow c\left(q_{j}, x, y, s(z)\right)\right\} \\
& \text { (e2) }\left[q_{i}, 2,-, q_{j}\right]: R_{M}:=R_{M} \cup\left\{f_{i}\left(c\left(q_{i}, x, s(y), z\right)\right) \rightarrow c\left(q_{j}, x, y, s(z)\right)\right\}
\end{aligned}
$$

Lemma 4.1. Given a reversible deterministic 2-counter Minsky machine $M$, the system $R_{M}$ has no RHS overlaps.

Proof. Suppose there is an RHS overlap between two rules in $R_{M}$. The only way for this to happen is for the rules' right hand sides to both be $c$-terms and to contain the same state $q_{i}$. Because $M$ is a reversible deterministic machine, and because we assume there is no quadruple with $q_{0}$ as its destination, the only way for that to happen is for one $c$-term to have been created by either transformation (a1) or (a2) (i.e., the quadruple in $\delta$ contained a $P$ ) and the other by the corresponding (b1) or (b2) (i.e., a $Z$ ). However, in that case, there can be no overlap, because there will be a function clash between $s$ and 0 . Thus there are no RHS overlaps.

Lemma 4.2. Given a reversible deterministic 2-counter Minsky machine $M$, the system $R_{M}$ is saturated by paramodulation and quasi-deterministic. 
Proof. That $R_{M}$ is saturated by paramodulation follows from the fact that none of the root symbols of the left hand sides of rules in $R_{M}$ occur at a position in the left- or right-hand side of any other rule. Therefore, there are no possible overlaps and $R_{M}$ is saturated trivially.

Recall that for the system $R_{M}$ to be quasi-deterministic, the following conditions must hold:

(1) $R_{M}$ has no root pair repetitions

(2) If $(s \rightarrow t) \in R_{M}$, then neither $t$ nor $s$ is a variable

(3) If $(s \rightarrow t) \in R_{M}$, then $\operatorname{root}(s) \neq \operatorname{root}(t)$

For each quadruple in $\delta$, only one of the above transformations will be used to extend $R_{M}$ (i.e., only one rule will be added). The only way two rules could have the same left-hand root symbol is for them to contain the same state $q_{i}$, and that is only possible if one was created by either transformation (a1) or (a2) (i.e., the quadruple in $\delta$ contained a $P$ ) and the other by the corresponding (b1) or (b2) (i.e., a $Z$ ). In that case, however, one would have $f_{i}$ as its left-hand root symbol, and the other would have $f_{i}^{\prime}$. Thus condition 1 is met.

Conditions 2 and 3 are readily apparent from the rules in $R_{M}$.

Lemma 4.3. Given a reversible deterministic 2-counter Minsky machine $M$, the system $R_{M}$ is convergent.

Proof. To prove that $R_{M}$ is terminating, consider the measure function

$$
\phi: T(\Sigma, \mathcal{X}) \rightarrow \mathbb{N} \times \mathbb{N}
$$

such that $\phi(t)=\left(n_{1}, n_{2}\right)$, where $n_{1}$ is the number of occurrences of $f, f_{i}$, and $f_{i}^{\prime}$ symbols for all $i$, and $n_{2}$ is the number of occurrences of $g$ and $g^{\prime}$ symbols. Note that every rule except

$$
f_{L}\left(c\left(q_{L}, s^{k^{\prime}}(0), s^{p^{\prime}}(0), z\right)\right) \rightarrow g\left(c\left(e^{\prime}, 0,0, z\right)\right)
$$

removes at least one of these symbols and does not add any of the others. For this remaining rule, the first component decreases even though the second component increases. Since the rules in $R_{M}$ are linear and variable-preserving, if $t_{1} \rightarrow_{R_{M}} t_{2}$, then $\phi\left(t_{1}\right)>_{\text {lex }} \phi\left(t_{2}\right)$, where $>_{\text {lex }}$ is the lexicographic order induced by $>$ on $\mathbb{N} \times \mathbb{N}$. Therefore $R_{M}$ is terminating.

We will show the confluence of $R_{M}$ by showing that $R_{M}$ has no critical pairs. As shown in Lemma 4.2 no two rules added by transformations (a1)-(e2) have the same root symbol on their left-hand sides. No rule's left-hand side's root symbol could occur at a non-root position of another rule's left-hand side. So the only possible overlap would be the third and fourth initial rules, but note that their left-hand sides are not unifiable due to a function clash of 0 and $s(z)$. Thus $R_{M}$ has no critical pairs. So $R_{M}$ is convergent.

Lemma 4.4. Given a reversible deterministic 2-counter Minsky machine $M$, configurations $\left(q_{0}, k, p\right)$ and $\left(q_{L}, k^{\prime}, p^{\prime}\right)$, and rewrite system $R_{M}$ constructed as above, $R_{M}$ is subterm-collapsing if and only if the machine $M$, starting in configuration $\left(q_{0}, k, p\right)$, will halt with $\left(q_{L}, k^{\prime}, p^{\prime}\right)$ as its final configuration.

Proof of 'if'. Suppose the machine $M$, starting in the given initial configuration, does halt with the given final configuration after $n$ steps. Since $M$ is deterministic, there is a single sequence of transitions the machine can take. Let $S=\left\langle\tau_{1}, \ldots, \tau_{n}\right\rangle$ be that sequence of transitions. For each $\tau_{i}=\left[q_{j}, \kappa, d, q_{j^{\prime}}\right]$, we define the symbol $f_{i}^{*}$ as

$$
f_{i}^{*}= \begin{cases}f_{j}^{\prime} & \text { if } d=Z \\ f_{j} & \text { otherwise }\end{cases}
$$


In other words, $f_{i}^{*}$ is an alias for either $f_{j}^{\prime}$ or $f_{j}$ depending on how $M$ left state $q_{j}$.

Now we can construct a term $t=f_{L}\left(\left(f_{n}^{*} \circ \cdots \circ f_{1}^{*}\right)\left(f\left(c\left(e, s^{k}(0), s^{p}(0), 0\right)\right)\right)\right)$, where $\circ$ is function composition, such that $t \rightarrow_{R_{M}}^{+} g\left(c\left(e^{\prime}, 0,0, s^{n}(0)\right)\right)$. This should be apparent from the definition of $R_{M}$. After the first rule initializes the machine and removes the $f$, each rewrite will remove the innermost $f_{i}^{*}$ and move to the next configuration. Finally the second rule will remove the outermost $f_{L}$ and move to the empty state.

Finally we can create a term $t^{\prime}=\left(g^{\prime} \circ g\right)^{n}\left(g^{\prime}(t)\right)$ such that $t^{\prime} \rightarrow_{R_{M}}^{+} e$. This is because each application of the third rule will remove the innermost $\left(g^{\prime} \circ g\right)$ and an $s$ from the step counter, until the counter becomes 0 . At that point, the fourth rule will rewrite the entire term to $e$. Note that $e$ is a proper subterm of $t$, which is itself a subterm of $t^{\prime}$, so $R_{M}$ is subterm-collapsing.

Proof of 'only if'. Conversely, suppose $R_{M}$ is subterm-collapsing. Since all rewrite rules in $R_{M}$ are variable-preserving, and each rule added by transformations (a1)-(e2) increases the step counter in the $c$-term, each application of a rule creates a $c$-term which has never occurred before. Therefore, the only way for a subterm-collapse to occur is for a term to rewrite to $e$ by the fourth rule.

Let $t$ be a term with $e$ as a proper subterm such that $t \rightarrow_{R_{M}}^{+} e$. The only way to rewrite $t$ is by the first rule. This means there is a position $p$ in $t$ such that $\left.t\right|_{p}=f\left(c\left(e, s^{k}(0), s^{p}(0), 0\right)\right)$. Therefore, there is a term $t^{\prime}$ such that $t \rightarrow_{R_{M}} t^{\prime}$ and $\left.t^{\prime}\right|_{p}=c\left(q_{0}, s^{k}(0), s^{p}(0), 0\right)$.

At this point, the only way to rewrite $t^{\prime}$ is to use the rules added by transformations (a1)(e2). Therefore, there must be a sequence $S=\left\langle i_{1}, \ldots, i_{n}\right\rangle, 0 \leq i_{j}<L$, and a position $p^{\prime}$ in $t$, $p^{\prime}<p$, such that $\left.t\right|_{p^{\prime}}=\left.t^{\prime}\right|_{p^{\prime}}=\left(f_{i_{n}}^{*} \circ \cdots \circ f_{i_{1}}^{*}\right)\left(\left.t\right|_{p}\right)$, where each $f_{j}^{*}$ is either $f_{j}$ or $f_{j}^{\prime}$. Therefore, there is a term $t^{\prime \prime}$ such that $t \rightarrow_{R_{M}} t^{\prime} \rightarrow_{R_{M}}^{+} t^{\prime \prime}$ and $\left.t^{\prime \prime}\right|_{p^{\prime}}=c\left(q_{L}, s^{k^{\prime}}(0), s^{p^{\prime}}(0), s^{n}(0)\right)$. It should be clear at this point, due to the way the rules of $R_{M}$ are constructed, that if the machine $M$ is started in configuration $\left(q_{0}, k, p\right)$, it would move through the states indexed by the sequence $S$, finally halting in configuration $\left(q_{L}, k^{\prime}, p^{\prime}\right)$.

Theorem 4.5. It is undecidable, given a rewrite system $R$ which is convergent, saturated by paramodulation, and for which RHS $R$ ) is quasi-deterministic, whether RHS $(R)$ is subtermcollapsing.

Proof. As shown in Lemma 4.4 the halting problem for reversible deterministic 2-counter Minsky machines can be reduced to checking for subterm-collapse in a rewrite system $R_{M}$. Since $R_{M}$ has no RHS overlaps (Lemma 4.1), $R H S\left(R_{M}\right)=R_{M}$. By Lemma 4.2, $R_{M}$ is saturated by paramodulation and quasi-deterministic, and by Lemma $4.3, R_{M}$ is convergent. Therefore, since the halting problem for reversible deterministic 2-counter Minsky machines is undecidable, so is checking for subterm-collapse in such a rewrite system.

\section{Conclusion and Future Work}

In this paper, we provesd that relaxing any of the Lynch-Morawska conditions can give rise to unification problems that are in $N P$ (or are not even decidable!). In doing so, we answered an open problem posed in the Lynch-Morawska paper [7. We also extended previous work [4] on undecidability of subterm-collapse to the (very) restricted term rewriting systems considered in this paper. So while the Basic Syntactic Mutation algorithm guarantees a polynomial time unification algorithm for term rewriting systems that fit its conditions, determining whether a system has these properties cannot be automated. 
Our future work involves extending the unification algorithm given in [7] to solve the asymmetric unification problem for theories which satisfy the Lynch-Morawska conditions. This type of unification problem has only recently been studied and has applications in cryptographic protocol analysis [5].

\section{References}

[1] S. Anantharaman, S. Erbatur, C. Lynch, P. Narendran, and M. Rusinowitch. Unification Modulo Synchronous Distributivity. In B. Gramlich, D. Miller, and U. Sattler, editors, IJCAR, volume 7364 of Lecture Notes in Computer Science, pages 14-29. Springer, 2012.

[2] F. Baader and T. Nipkow. Term Rewriting and All That. Cambridge University Press, 1999.

[3] F. Baader and W. Snyder. Unification Theory. In Alan Robinson and Andrei Voronkov, editors, Handbook of Automated Reasoning, pages 440-526. Elsevier Science Publishers BV, 1999.

[4] H.J. Bürckert, A. Herold, and M. Schmidt-Schauss. On Equational Theories, Unification, and (Un)Decidability. Journal of Symbolic Computation, 8(1):3-49, 1989.

[5] S. Erbatur, S. Escobar, D. Kapur, Z. Liu, C. Lynch, C. Meadows, J. Meseguer, P. Narendran, S. Santiago, and R. Sasse. Effective Symbolic Protocol Analysis via Equational Irreducibility Conditions. In Sara Foresti, Moti Yung, and Fabio Martinelli, editors, ESORICS, volume 7459 of Lecture Notes in Computer Science, pages 73-90. Springer, 2012.

[6] M. Garey and D. Johnson. Computers and Intractability: A Guide to the Theory of NPCompleteness. W. H. Freeman \& Co., New York, NY, USA, 1979.

[7] C. Lynch and B. Morawska. Basic Syntactic Mutation. In A. Voronkov, editor, CADE, volume 2392 of Lecture Notes in Computer Science, pages 471-485. Springer, 2002.

[8] K. Morita. Universality of a reversible two-counter machine. Theoretical Computer Science, 168(2):303-320, 1996.

[9] E. Ohlebusch. Termination is not Modular for Confluent Variable-Preserving Term Rewriting Systems. Inf. Process. Lett., 53(4):223-228, 1995. 


\section{A Forward Closure}

In an earlier version of the paper, we formulated the Lynch-Morawska conditions in terms of the concept of forward closure. The forward closure of a convergent term rewriting system $R$ is defined in terms of the following operation on rules in $R$ : Let $\rho_{1}=\left(l_{1} \rightarrow r_{1}\right)$ and $\rho_{2}=\left(l_{2} \rightarrow r_{2}\right)$ be two rules in $R$ and let $p \in \mathcal{F} \mathcal{P}$ os $\left(r_{1}\right)$. Then

$$
\rho_{1} \rightsquigarrow p \rho_{2}=\sigma\left(l_{1} \rightarrow r_{1}\left[r_{2}\right]_{p}\right)
$$

where $\sigma=m g u\left(\left.r_{1}\right|_{p}=? l_{2}\right)$.

Given rewrite systems $R_{1}, R_{2}$, and $R_{3}$ we define $\mathcal{F} \mathcal{O V}\left(R_{1}, R_{2}\right)$ (forward overlap) and $\mathcal{N}\left(R_{1}, R_{2}, R_{3}\right)$ (non-redundant) as

$$
\begin{aligned}
\mathcal{F} \mathcal{O V}\left(R_{1}, R_{2}\right) & =\left\{\rho_{1} \rightsquigarrow_{p} \rho_{2} \mid \rho_{1}=\left(l_{1} \rightarrow r_{1}\right) \in R_{1}, \rho_{2} \in R_{2}, \text { and } p \in \mathcal{F P} \text { os }\left(r_{1}\right)\right\} \\
\mathcal{N}\left(R_{1}, R_{2}, R_{3}\right) & =\left\{\rho \mid \rho \in \mathcal{F} \mathcal{O V}\left(R_{1}, R_{2}\right) \text { and } \rho \text { is not redundant in } R_{3}\right\}
\end{aligned}
$$

We now simultaneously define $N R_{k}(R)$ (new rules) and $F C_{k}(R)$ (forward closure) for all $k \geq 0$.

$$
\begin{array}{ll}
N R_{0}(R)=R & N R_{k+1}(R)=\mathcal{N}\left(N R_{k}(R), R, F C_{k}(R)\right) \\
F C_{0}(R)=R & F C_{k+1}(R)=F C_{k}(R) \cup \mathcal{N}\left(N R_{k}(R), R, F C_{k}(R)\right)
\end{array}
$$

Finally,

$$
F C(R)=\bigcup_{i=1}^{\infty} F C_{i}(R)
$$

Note that $F C_{j}(R) \subseteq F C_{j+1}(R)$ for all $j \geq 0$. A set of rewrite rules $R$ is forward-closed if and only if $F C(R)=R$.

However it turns out that:

Lemma A.1. It is not the case that every convergent, forward-closed term rewriting system is saturated by paramodulation.

Proof. Consider the following term rewriting system $R$ :

$$
\begin{aligned}
f(g(x, x)) & \rightarrow h(x, x) \\
g(s(x), s(y)) & \rightarrow h(s(x), s(y)) \\
f(h(x, x)) & \rightarrow h(x, x)
\end{aligned}
$$

The only nontrivial critical pair is between the first and second rules. This pair is given as follows:

$$
\langle f(h(s(u), s(v))), h(s(u), s(v))\rangle
$$

The equation formed by this pair, $f(h(s(u), s(v))) \approx h(s(u), s(v))$, is not redundant, since $h(s(u), s(v))$ is in normal form and the only way to join the terms is by the third rule. The instance $f(h(s(u), s(v))) \rightarrow h(s(u), s(v))$ is equal to the equation, and thus not lower in the ordering. 